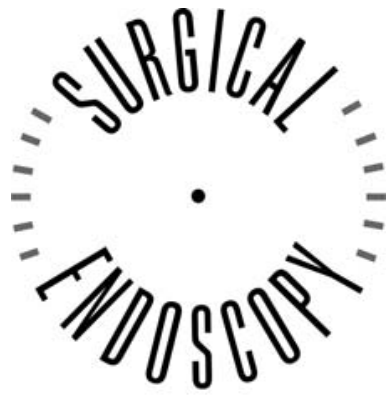

and Other Interventional Techniques

\title{
The use of autologous platelet-leukocyte gels to enhance the healing process in surgery, a review
}

\author{
P. A. Everts, ${ }^{1}$ E. P. Overdevest, ${ }^{1}$ J. J. Jakimowicz, ${ }^{2}$ C. J. Oosterbos,${ }^{3}$ J. P. Schönberger, ${ }^{4}$ J. T. Knape, ${ }^{6}$ \\ A. van Zundert ${ }^{5}$ \\ ${ }^{1}$ Department of Perioperative Blood Management, Catharina Hospital Eindhoven, Michelangelolaan 2, 5623 EJ, Eindhoven, The Netherlands \\ ${ }^{2}$ Department of General Surgery, Catharina Hospital Eindhoven, Michelangelolaan 2, 5623 EJ, Eindhoven, The Netherlands \\ ${ }^{3}$ Department of Orthopaedic Surgery, Catharina Hospital Eindhoven, Michelangelolaan 2, 5623 EJ, Eindhoven, The Netherlands \\ ${ }_{5}^{4}$ Department of Cardiothoracic surgery, Catharina Hospital Eindhoven, Michelangelolaan 2, 5623 EJ, Eindhoven, The Netherlands \\ ${ }^{5}$ Department of Anaesthesiology, Catharina Hospital Eindhoven, Michelangelolaan 2, 5623 EJ, Eindhoven, The Netherlands \\ ${ }^{6}$ Department of Anaesthesiology, University Medical Centre, Utrecht, The Netherlands
}

Received: 27 November 2006/Accepted: 22 December 2006/Online publication: 13 April 2007

\begin{abstract}
Background: The therapeutic use of autologously prepared, platelet-leukocyte-enriched gel (PLG) is a relatively new technology for the stimulation and acceleration of soft tissue and bone healing. The effectiveness of this procedure lies in the delivery of a wide range of platelet growth factors mimicking the physiologic wound healing and reparative tissue processes. Despite an increase in PLG applications, the structures and kinetics of this autogenously derived biologic material have not been observed.

Methods: A review of the most recent literature was performed to evaluate the use of PLG in various surgical disciplines.

Results: The review showed that the application of PLG has been extended to various surgical disciplines including orthopedics, cardiac surgery, plastic and maxillofacial surgery, and recently also endoscopic surgery.

Conclusion: This review demonstrates the usefulness of PLG in a wide range of clinical applications for improvement of healing after surgical procedures.
\end{abstract}

Key words: Growth factors - Leukocytes - Platelet gel - Wound healing

Soft tissue wound healing and bone growth involve physiologic cascades in which cellular and hormonal factors play pivotal roles $[1,2]$. Some of these cascade components can be isolated from autologously drawn whole blood. Point-of-care devices can intraoperatively fractionate the autologous blood into platelet-poor plasma, platelet-leukocyte-rich plasma (P-LRP), and red blood cells $[3,4]$. The P-LRP fraction, a mixture of concentrated platelets and leukocytes, can be activated by (autologous) thrombin to create a viscous solution known as platelet-leukocyte gel (PLG). This platelet coagulum can be exogenously applied to soft wound tissues, bone, or synthetic bone as a spray or as a solid, clotted, gelatinous mass. The rationale for applying platelet gel is based on the delivery of platelet growth factors to tissues and on the fact that platelet $\alpha$-granules, found inside the platelets, contain a variety of growth factors [5]. Platelet gel growth factors are peptides that promote cell proliferation, differentiation, chemotaxis, and the migration of various cells involved in both wound healing and bone growth $[6,7]$.

Recently, numerous P-LRP devices have become available for therapeutic use to stimulate and accelerate soft tissue and bone healing and to control postoperative wound bleeding. The rationale for applying PLG lies in the mimicking and accelerating of physiologic wound healing and reparative tissue processes.

This article provides information on the results of electron microscopic imaging used to evaluate the content of PLG. Furthermore, because the use of PLG is a relatively new per- and/or perioperative biotechnological application, new and additional indications for the use of PLG are defined. New PLG applications are reviewed for different applications including cardiac surgery, general surgery, orthopedics and traumatology, cosmetic surgery, maxillofacial surgery, sports medicine, and endoscopic surgical procedures.

\section{Defining platelet-leukocyte-rich gel}

Platelet-leukocyte gel is prepared from a high concentration of platelets acquired from freshly drawn autologous whole blood. In general, this blood is obtained by 

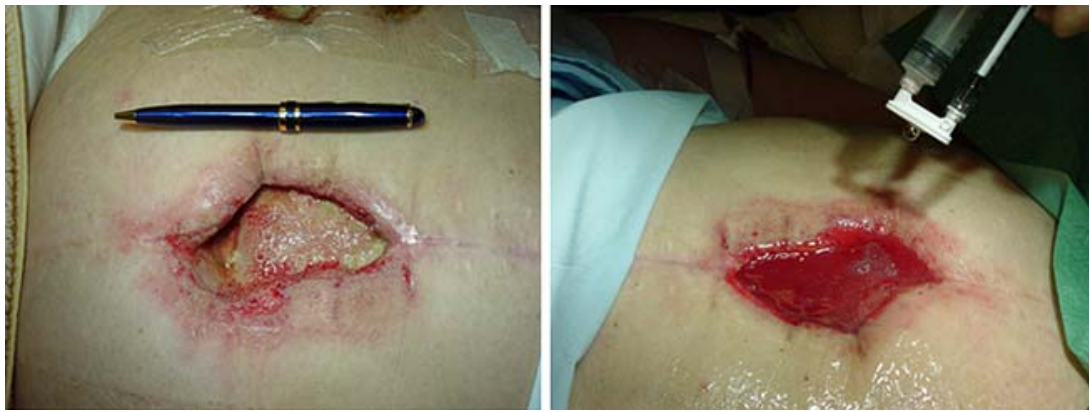

Fig. 1. Using a double-syringe delivery technique, platelet-leucocyte gel is applied on the skin wound of a patient with decubitis ulceration.

point-of-care devices and sequestered into different blood components, namely, P-LRP, platelet-poor plasma, and erythrocyte concentrate [8].

One characteristic of P-LRP is that it comprises a small volume of plasma with fibrinogen, platelets, and leukocytes. Platelets suspended in the P-LRP are in an inactive state and in liquid form. The platelet growth factors are found inside $\alpha$ granules, which are present in the platelet cytoplasm. Platelets become immediately activated because of interaction with thrombin, the most potent platelet activator, and a sticky platelet aggregate is formed. Subsequently, platelet $\alpha$ granules release growth factors into the extracellular environment, where they bind to specific platelet growth factor receptors. After this, through intracellular tissue signaling, a number of pathways are triggered that initiate the healing process.

Besides a high concentration of platelets in PLG, several differentiated and nonactivated leukocytes are present at high levels. In particular, these include neutrophilic granulocytes and monocytes, both known for host defense mechanism actions against bacteria through the action of myeloperoxidase that creates hypochloric acid. The number of platelets in PLG is three to seven times higher than normally found, whereas the white blood cell count is two to four times greater than normal [9-11].

\section{Healing mechanisms of platelet-leukocyte gels}

It is generally accepted that platelet growth factors play a central role in the healing process and tissue formation [12]. Wound healing is a well-orchestrated and complex series of events involving cell-cell and cell-matrix interactions, in which platelet growth factors serve as messengers to regulate various processes. Initially, tissue repair begins with platelet clot formation, activation of the coagulation cascade and platelet degranulation, and the release of growth factors. After this release, specific platelet growth factors bind to particular target tyrosine growth factor receptors, which subsequently activate intracellular signal transduction pathways [7, 13].

During the first 2 days of wound healing, an inflammatory process is initiated by migration of neutrophils and subsequently macrophages to the wound site. In turn, activated macrophages release multiple growth factors, including platelet-derived growth factor, transforming growth factors alpha and beta, interleukin-1, and fibroblast growth factor [11]. Angiogenesis and fibroplasia start shortly after day 3 , followed by collagen synthesis on days 3 to 5 . This process leads to an early increase in wound-breaking strength, which is the most important wound-healing parameter of surgical wounds, followed by epithelialization and the ultimate remodeling process [14].

Some functions of platelet growth factors in tissue repair showed that a controlled sequential appearance is crucial after primary wound closures at the end of surgical procedures for the treatment of bone and cartilage defects as well as muscle and tendon lesions, and for the promotion of synthetic tissue ingrowth during reconstructive surgeries [15].

Based on the actions of the various platelet growth factors during the different stages in the wound healing cascade, the use of autologous PLG to stimulate wound repair is an interesting proposition, although recombinant growth factors have been used to stimulate wound healing. However, as compared with recombinant single growth factor applications, platelet gels have the supreme advantage in that they synergistically induce various growth factors and promote mitogenesis of mesenchymal stem cells at the wound site $[16,17]$.

\section{Novel platelet-leukocyte gel applications}

After the activation of P-LRP with thrombin, a viscous gel with a degree of plasticity is formed that sticks to wound tissues. At this stage, PLG is exogenously applied, using a syringe delivery technique, to surgical wound sites during closure and to soft tissue structures to stimulate tissue regeneration (Fig. 1a and b), , or it is mixed with bone or bone substitutes to accelerate bone healing.

The outcome of multiple studies on the efficacy of PLG treatment have been published [18-20]. Proponents of PLG application refer to improved wound healing and increased bone growth to warrant its use. A reduction in the development of severe postoperative wound infections with the application of PLG during incisional wound closure after a cardiac surgical procedure has been reported [21]. These observations indicate that this is a promising technique, with the result that the delivery of autologous platelet growth factors and vital neutrophilic leukocytes is now gaining more popularity. However, more scientific evidence and data to support the use of PLG in clinical settings are mandatory for progress in the use of these autologous bio- 


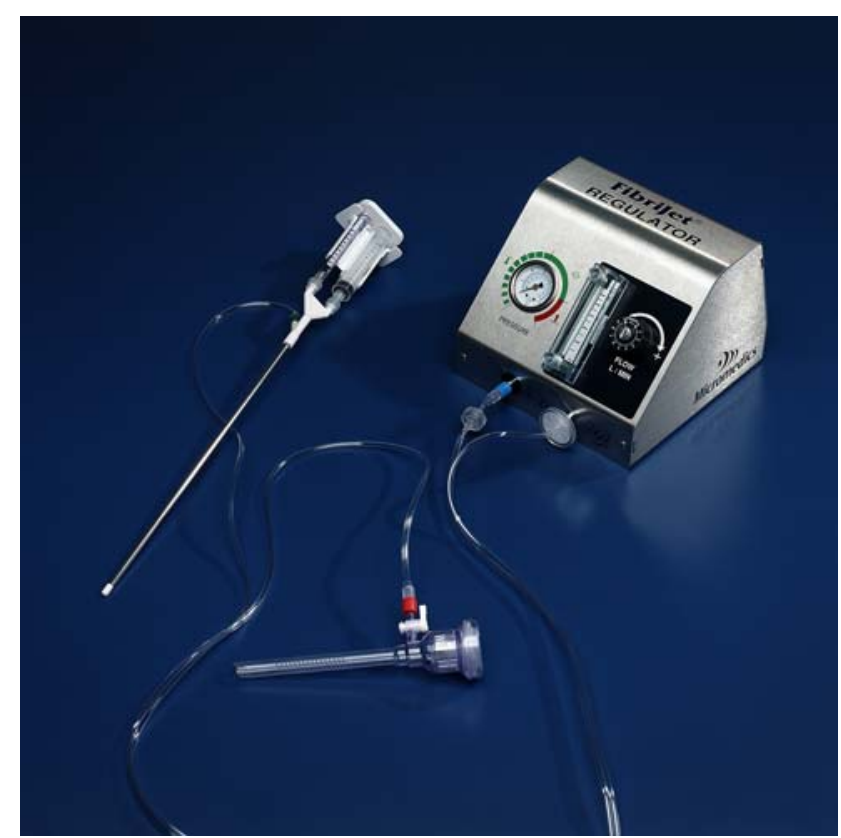

Fig. 2. Aerosol-controlled laparascopic platelet-leukocyte gel delivery system.

technology procedures to be achieved. Recently, novel applications have emerged in the field of PLG applications and in a variety of surgical disciplines. We reviewed some of these new applications and report on some results of studies that indicate some potentially new directions and clinical applications.

\section{Soft tissue healing and endoscopic applications}

Promising results have been obtained for patients with chronic nonhealing (diabetic) wounds after topical PLG application. Margolis et al. [22] demonstrated that the application of PLG was more effective than standard care methods for wound healing, and that this treatment was even more effective for patients with deeper wounds. Furthermore, in our own experience we have encountered improved wound healing with the application of PLG during wound closure after total knee arthroplasty [23].

Soft tissue trauma such as tendon and ligament ruptures and joint capsular injuries, which frequently occur, often require a surgical intervention [24, 25]. It is presumed that surgical repair combined with the application of biologically active PLG should accelerate healing with an improved outcome. Aspenberg and Virchenko [26] showed, in an in vivo rat model, that PLG applied to traumatized Achilles tendons increased tensile strength and stiffness by about $30 \%$ after the first week. The effect persisted for as long as 3 weeks after the injection, suggesting that the use of PLG in tendon repair improved the physiologic healing process.

Anterior cruciate reconstructive ligament surgery is routinely performed to reconstruct the ligament with an autologous graft. Because the procedure is mainly performed arthroscopically, it is challenging to apply PLG to augment healing. Sanchez et al. [27] reported en- hanced healing with fewer complications and improved fixation of the graft within the bone tunnels in a retrospective clinical trial involving 100 patients.

The mechanism of action by which tendon repair is improved with the application of PLG is based on release of vascular endothelial growth factor (VEGF). This growth factor stimulates angiogenesis, leading to an improved blood supply, which is mandatory for the tendon repair process [28]. Furthermore, released platelet growth factors induce a proliferation of tendon cells and stimulate production of VEGF and hepatocyte growth factor, a potent antifibrotic agent. The latter may be of importance in reducing scar formation and fibrosis in newly reconstructed tendon tissue, which may lead to poor outcomes [29].

In the past, fibrin glues have been used to make a sutureless intestine anastomosis and to treat gastrointestinal anastomotic leaks during laparoscopic surgery $[30,31]$.

Recently, a novel PLG application has emerged for morbidly obese patients undergoing bariatric surgery. Brady et al. [32] used PLG via an endoscopic delivery system (Fig. 2), after a laparoscopic Roux-en Y gastric bypass procedure to avoid hemorrhage, infection, and anastomotic leaks, which may occasionally lead to death. These authors suggest that the use of PLG contributed to an enhanced hemostatic response, accelerated tissue healing, and improved collagen synthesis, thus preventing anastomotic leaks and thereby improving outcome. Recently, Pomerantz and Dutton [33] applied PLG during endoscopic sinus surgery to improve their packing technique. Their quality-of-life scores showed an improvement in terms of postoperative epistaxis, synechia formation, and exuberant granulation tissue in PLG-treated patients.

\section{Synthetic and allogeneic implants}

Synthetic meshes and allogeneic implants are currently used in "tension-free" hernioplasty of the abdominal wall and in inguinal hernia repair [34]. However, after cicatricial hernia repair, serious complications have been observed (i.e., seromata, dense adhesions, fistulization). Furthermore, Tyrell et al. [35] observed that the tensile strength of implanted meshes was markedly reduced due to absorption of the mesh. Lichtenstein hernioplasty is an accepted method for inguinal hernia repair. One of the most frequent complications after inguinal hernia surgery is postoperative pain, which at times is chronic and permanent, leading to a poor quality of life [36, 37]. Innovative tools for decreasing the complication related to anchoring of the mesh in position include the use of tissue adhesives [38]. Another option for securing the synthetic implants may be realized by applying PLG to the meshes rather than to full suture lines. Autologous platelet growth factors have been used by Zieren et al. [39], who showed enhanced ingrowth and increased cell proliferation with a higher number of fibroblasts/collagen fibers in abdominal hernia repair, suggesting a role of platelet growth factors in the healing process. Sclafani et al. [40] showed an accelerated maturation of wounds 
in an experimental setting when PLG was used. On the basis of these findings, we have initiated a research program to confirm whether the application of PLG is useful for hernia repair in a clinical setting. The study objective is to determine the advantages of PLG use as compared with current techniques, especially in minimizing pain after conventional and laparoscopic hernioplasties.

\section{Bone growth}

Impaired bone healing after fractures, with the development of pseudarthrosis, or fusion operations in the case of nonunions cause pain and disability. Attempts are being made to create bone substitutes, but technologies are being developed that improve bone healing by adding biologic materials, such as PLG, to stimulate osteogenesis and osteoconduction. In bone healing (i.e., callus formation), platelets act as an exogenous source of growth factors stimulating the activity of bone cells on the basis of a unique role in bone growth [41, 42].

Numerous in vitro studies using platelet gels have been performed. Since the initial description of PLG use in maxillofacial surgery, subsequent studies have focused on the effect from a variety of bone-derived cells including osteoblasts, osteoclasts, periodontal ligament cells, and mesenchymal cells. Gruber et al. [43] demonstrated that proliferation of bone-derived cells was augmented, in a dose-dependent manner, when PLG was used. They suggested that, aside from a mitogenic effect, PLG application at the time of surgery enhances bone healing capacity due to bone resorption and remodeling.

Another therapeutic application of PLG involves the combination of PLG with different bone substitutes. Several authors have used histomorphometric analysis to demonstrate a beneficial effect of PLG when different bone matrices are used. Aghaloo et al. [44] used natural deproteinized bovine matrix and measured improved bone growth when it was used with PLG. Suba et al. [45] used $\beta$-tricalcium phosphate in combination with PLG and observed more intense bone regeneration. Furthermore, due to the sticky structure of PLG, caused by the fibrin strands present in the gel, bone substitutes are kept together, avoiding unwanted migration of bone particles.

Recently, the percutaneous application of PLG in a diabetic femur fracture model was described. Normalized cellular proliferation and chondrogenesis, with improved mechanical strength, were observed when PLG was injected in this model [46].

\section{Tissue engineering}

A variety of methods have been used for the restoration of bone or soft tissue defects in different surgical settings, including orthopedic surgery, maxillofacial surgery, and reconstructive surgery [47, 48]. However, the manipulation and reinforcement of biocompatible materials in surgery is not always easy to achieve.
Mixtures of autologous tissues have been used to accomplish restoration of defects. Tissue engineering, a technology involving the morphogenesis of new tissues using isolated cells with biocompatible matrices, often is combined with growth factors. Mesenchymal stem cells (MSC) are multipotent cells that can replicate as undifferentiated cells with the possibility of differentiating into mesenchymal tissues (i.e., bone, cartilage, tendon, muscle). This ability has made MSC a potential component of tissue engineering concepts. Recently, several research groups have been studying PLG as a matrix for tissue-engineering models because the activated PLG releases numerous platelet-derived growth factors [49, 50].

Another advantage of using MSC and PLG is that these two components are autologous, nontoxic, and biodegradable, proportional to the development of new bone formation, as compared with allogeneic matrices, which are nondegradable during the first weeks after implantation [51].

Yamada et al. [52] demonstrated in dogs that the combination of MSC and PLG resulted in a significantly higher maturation of bone and neovasularization than observed in control subjects. Thereafter, the same group used a combination of MSC and PLG for successful clinical alveolar bone augmentation [47]. The clinical application of PLG with bone marrow-derived MSC also was reported by Kito et al. [53] in the course of distraction osteogenesis. In soft tissue engineering, Anitua et al. [54] identified the role of PLG releasate on cultured tendon cells. These authors state that the treatment of tendon injuries may be of benefit because cell proliferation is induced with the promotion of endogenous angiogenic growth factor synthesis.

\section{Infection prevention}

Many investigators have focused on the exogenous application of concentrated and activated platelets in PLG for a variety of procedures that result in the material adhering to tissues, thereby initiating and accelerating wound healing [17, 22]. However, in addition to the platelet gel delivery of growth factors, limited data are available that address the role of leucocytes present in PLG to act as an antimicrobial component [10]. From our own experience, we have reported that PLRP not only comprises a high concentration of platelets containing platelet growth factors, but that it also is abundant in concentrated leukocytes, neutrophils, monocytes, and lymphocytes [11]. Neutrophils and monocytes are rich in granules containing myeloperoxidase, which catalyzes the oxidation of chloride to generate hypochlorous acid and other reactive oxygen derivates that act as potent bactericidal oxidants toxic to microorganisms and fungi $[55,56]$.

Furthermore, Yeaman et al. [57] and Tang et al. [58] support the idea that platelets also are involved in microbicidal activity, suggesting that they play a role in the platelet host defense mechanism by releasing a variety of platelet microbicidal proteins. The platelet microbicidal proteins were shown to be released after platelet acti- 
vation, demonstrating potent activities against pathogens that have a tendency to enter the bloodstream [59].

Furthermore, we expect that exogenous PLG injections rather than periarticular injections with corticosteroids or even surgery will be indicated for the treatment of tendonitis and periarthritis. Such a therapeutic approach was cautiously suggested in an equine study that applied PLG to injured tendons [60].

\section{Conclusion}

Platelet-leukocyte gels may become an ideal autologously prepared biologic blood-derived product that can be exogenously applied to a diversity of tissues, in which it releases high concentrations of platelet growth factors that enhance healing. In addition, it possesses antimicrobial properties that may contribute to the prevention of infections.

The current review suggests that the use of PLG may be beneficial in surgery. Platelet-leukocyte gels have been successfully used in maxillofacial surgery, orthopedics, cosmetic surgery, and dental implantology. However, the procedure for preparing PLG and the techniques of application are likely to differ greatly among clinicians, resulting in inconsistent results. To avoid conflicting data, standardization of P-LRP methodology is therefore warranted. Furthermore, randomized controlled clinical trials are needed to study the effect of PLG on wound rehabilitation, functional recovery, and the promotion of bone growth. The bactericidal effect of PLG should be clarified, and its role in tissue engineering should be defined. Overall, it may be concluded that the structures present in PLG appear to have a major beneficial therapeutic effect in surgery.

\section{References}

1. Hunt TK (1990) Basic principles of wound healing. J Trauma 30(Suppl): 122-128

2. Giannoble WV (1996) Periodontal tissue engineering by growth factors. Bone 19(Suppl): 23-37

3. Landesberger R, Moses M, Karpatkin M (1998) Risks of using platelet rich plasma. J Oral Maxillofac Surg 56: 1116-1117

4. Weibrich G, Kleis WKG, Hafner G (2002) Growth factor levels in the platelet-rich plasma produced by 2 different methods: curasantype PRP kit versus PCCS PRP system. Int J Oral Maxillofac Imp 17: $184-190$

5. Zucker-Franklin C (1989) The relationship of alpha granules to the membrane system of platelets and megakaryocytes. Blood Cells 15: 73-79

6. Slater M, Patava J, Kingham K, Mason RS (1995) Involvement of platelets in stimulating osteogenic activity. J Orthop Res 13: 655663

7. Tabata Y (2003) Tissue regeneration based on growth factor release. Tissue Eng 9(Suppl): 5-15

8. Kevy SV, Jacobson MS (2004) Comparison of methods for point of care preparation of autologous platelet gel. J Extra Corpor Technol 36: 28-35

9. Everts PAM, Brown Mahoney Ch, Hoffmann JJHL, Schönberger JPAM, Box HAM, van Zundert A, Knape JTA (2006) Plateletrich plasma preparation using three devices: implications on platelet activation and platelet growth factor release. Growth Factors 24: 164-171
10. Fréchette J-P, Martineau I, Gagnon G (2005) Platelet-rich plasmas: Growth factor content and roles in wound healing. J Dent Res 84: 434-439

11. Everts PAM, Hoffmann JJHL, Weibrich G, Brown Mahoney Chr, Schönberger JPAM, van Zundert A, Knape JTA (2006) Autologous platelet gel growth factor release and leukocyte kinetics using three devices. Transfus Med 16: 363-368

12. Werner S, Grose R (2003) Regulation of wound healing by growth factors and cytokines. Physiol Rev 83: 835-870

13. Lieberman JR, Daluiski A, Einhorn TA (2002) The role of growth factors in the repair of bone. J Bone Joint Surg Am 84: 1032-1044

14. Robson MC (1991) Growth factors as wound healing agents. Curr Opion Biotechnol 2: 863-867

15. Luginbuehl V, Meinel L, Merkle HP, Gander B (2004) Localized delivery of growth factors for bone repair. Eur J Pharm Biopharm 58: $197-208$

16. Lynch SE, Nixon JC, Colvin RB, Antoniades HN (1987) Role of platelet-derived growth factor in wound healing: synergistic effects with other growth factors. Proc Natl Acad Sci U S A 84: 76967700

17. Brown RL, Breeden MP, Greenhalg DG (1994) PDGF and TGFalpha act synergistically to improve wound healing in the genetically diabetic mouse. J Surg Res 56: 562-570

18. Man D, Plosker H, Winland-Brown JE (2001) The use of autologous platelet-rich plasma [platelet gel] and autologous plateletpoor plasma [fibrin glue] in cosmetic surgery. Plast Reconstr Surg 107: 229-237

19. Marx RE, Carlson ER, Eichstaedt RM, Schimmele SR, Strauss JE, Georgeff KR (1998) Platelet-rich-plasma: growth factor enhancement for bone grafts. Oral Surg Oral Med Oral Pathol Oral Radiol Endod 85: 638-646

20. Sanchez AR, Sheridan PJ, Kupp LI (2003) Is platelet-rich plasma the perfect enhancement factor? A current review. Int J Oral Maxillofac Surg 18: 93-103

21. Trowbridge CC, Stammers AH, Woods E, Yen BR, Klayman M (2005) Use of platelet gel and its effects on infection in cardiac surgery. J Extra Corpor Technol 37: 381-386

22. Margolis DJ, Kantor J, Santanna J, Strom BL, Berlin JA (2001) Effectiveness of platelet releasate for the treatment of diabetic neuropathic foot ulcers. Diabetes Care 24: 483-488

23. Everts PAM, Devilee RJJ, Brown-Mahoney Chr, Eeftinck Schattenkerk M, Box HAM, Knape JTA, van Zundert A (2006) Platelet gel and fibrin sealant reduce allogenic blood transfusions and in total knee arthroplasty. Acta Anaesth Scand 50: 593-599

24. Kannus P, Natri A (1997) Etiology and pathophysiology of tendon ruptures in sports. Scan J Med Sci Sports 7: 107-112

25. Sharma P, Mafulli N (2005) Tendon injury and tendinopathy: healing and repair. J Bone Joint Surg Am 87: 187-202

26. Aspenberg P, Virchenko O (2004) Platelet concentrate injection improves Achilles tendon repair in rats. Acta Orthop Scand 75: 93-99

27. Sanchez M, Anitua E, Andia I (2003) Use of autologous plasmarich in growth factors in arthroscopic surgery. Cuader Artroscopia 10: $12-19$

28. Anitua E (2006) Reciprocal actions of platelet-secreted TGF-b1 on the production of VEGF and HGF by human tendon cells. Plast. Reconstr Surg (in press)

29. Awad HA, Boivin GP, Dressier MR, Smith FN, Young RG, Butler DL (2003) Repair of patellar tendon injuries using a cellcollagen composite. J Orthop Res 21: 420-431

30. Detweiler MB, Detweiler JG, Fenton J (1999) Sutureless and reduced suture anastomosis of hollow vessels with fibrin glue: a review. J Invest Surg 12: 245-262

31. Bonanomi G, Prince JM, McSteen F, Schauer PR, Hamad GG (2004) Sealing effect of fibrin glue on the healing of gastrointestinal anastomoses: implications for the endoscopic treatment of leaks. Surg Endosc 18: 1620-1624

32. Brady C, Vang S, Christensen K, Isler J, Vollstedt K, Holt D (2006) Use of platelet gel in bariatric surgery. J Extracorp Technol 38: 161-164

33. Pomerantz J, Dutton JM (2005) Platelet gel for endoscopic sinus surgery. Ann Otol Rhinol Laryngol 114: 699-704

34. Amid P, Shulman A, Lichtenstein I (1994) A critical evaluation of the Lichtenstein tension-free hernioplasty. Int Surg 79: 76-79 
35. Tyrell J, Silberman H, Chandrasoma P, Niland J, Shull J (1989) Absorbable versus permanent mesh in abdominal operations. Surg Gynecol Obstet 168: 227-232

36. Coderre TJ, Katz J, Vaccarino Al, MelzacK R (1993) Contribution of central neuroplasticity to pathological pain: review of clinical and experimental evidence. Pain 52: 259-285

37. Kawit R, Feichter A, Fuchsjager N, Kux M (1999) Postoperative pain and return to activity after five different types of inguinal herniorrhaphy. Hernia 3: 31-35

38. Canonico S, Santoriello A, Campitiello F, Fattopace A, Corte AD, Sordelli I, Benevento R (1999) Mesh fixation with human fibrin glue (Tissucol) in open tension-free inguinal hernia repair: a preliminary report. Hernia 9: 330-333

39. Zieren J, Castenholz E, Baumgart E, Muller JM (1999) Effects of fibrin glue and growth factors released from platelets on abdominal hernia repair with a resorbable PGA mesh: experimental study. J Surg Res 85: 267-272

40. Sclafani AP, Romo T, Ukrainsky G, McCormick SA, Litner J, Kevy SV, Jacobson MS (2005) Modulation of wound response and soft tissue ingrowth in synthetic and allogeneic implants with platelet concentrate. Arch Facial Plast Surg 7: 163-169

41. Bolander ME (1992) Regulation of fracture repair by growth factors. Proc Soc Exp Biol Med 200: 165-170

42. Thiede MA, Smock SL, Petersen DN, Grasser WA, Nishimoto SK, Thompson DD (1993) Production of osteocalcin by platelets: a potentially important link of platelet action in bone turnover. $\mathbf{J}$ Bone Miner Res 8(Suppl): 147-151

43. Gruber R, Karreth F, Fischer MB, Watzek G (2002) Plateletreleased supernatants stimulate formation of osteoclast-like cells through a prostaglandin/RANKLdependent mechanism. Bone 30: 726-732

44. Aghaloo T, Moy P, Freymiller E (2004) Evaluation of platelet-rich plasma in combination with an organic bovine bone in the rabbit cranium: a pilot study. Int J Oral Maxillofac Implants 19: 59-65

45. Suba Z, Takacs D, Gyulai-Gaal S, Kovacs K (2004) Facilitation of b-tricalcium phosphate-induced alveolar bone regeneration by platelet-rich plasma in beagle dogs: a histologic and histomorphometric study. Int J Oral Maxillofac Implants 19: 832-838

46. Gandhi A, Doumas C, O'Connor JP, Parsons J, Lin S (2006) The effects of local platelet rich plasma delivery on diabetic fracture healing. Bone 38: 540-546

47. Yamada Y, Ueda M, Hibi H, Nagasaka T (2004) Translational research for injectable tissue engineered bone regeneration using mesenchymal stem cells and platelet-rich plasma: from basic research to clinical case study. Cell Transplant 13: 343-355

48. Langer R, Vacanti J (1993) Tissue engineering. Science 260: 920926
49. Lucarelli E, Beccheroni A, Donati D, Sangiorgi L, Cenacchi A, Del Vento AM, Giardino R, Fornasari PM, Mercuri M, Picci P (2003) Platelet-derived growth factors enhance proliferation of human stromal stem cells. Biomaterials 24: 3095-3100

50. Zhu SJ, Choi BH, Jung JH, Lee SH, Huh JY, You TM, Lee HJ, Li J (2006) A comparative histologic analysis of tissue-engineered bone using platelet-rich plasma and platelet-enriched fibrin glue. Oral Surg Oral Med Oral Pathol Oral Radiol Endod 102: 175-179

51. Anitua E, Sanchez M, Nurden A, Nurden P, Orive G, Andia I (2006) New insights into and novel applications for platelet-rich fibrin therapies. Trend Biotechnol 24: 227-234

52. Yamada Y, Ueda M, Naiki T, Takahashi M, Hata K, Nagasaka T (2004) Autogenous injectable bone for regeneration with mesenchymal stem cells and platelet-rich plasma: tissue-engineered bone regeneration. Tissue Eng 10: 955-964

53. Kitoh H, Kitakoji T, Tsuchiya H (2004) Transplantation of marrow-derived mesenchymal stem cells and platelet-rich plasma during distraction osteogenesis: a preliminary result of three cases. Bone 35: 892-898

54. Anitua E, Andia I, Sanchez M, Azofra J, del Mar Zalduendo M, de la Fuente M, Nurden P, Nurden AT (2004) Autologous preparations rich in growth factors promote proliferation and induce VEGF and HGF production by human tendon cells in culture. $\mathrm{J}$ Orthop Res 23: 281-286

55. Hazen SL, d'Avignon A, Anderson MM, Hsu FF, Heinecke JW (1998) Human neutrophils employ the myeloperoxidase-hydrogen peroxide-chloride system to oxidize $\alpha$-amino acids to a family of reactive aldehydes. J Biol Chem 273: 4997-5005

56. Lincoln JA, Lefkowitz DL, Cain T, Castro A, Mills KC, Lefkowitz SS, Moguilevsky N, Bollen A (1995) Exogenous myeloperoxidase enhances bacterial phagocytosis and intracellular killing by macrophages. Infect Immun 63: 3042-3047

57. Yeaman MR, Bayer AS, Koo S-P, Foss W, Sullam PM (1998) Platelet microbicidal proteins and neutrophil defensin disrupt the Staphylococcus aureus cytoplasmic membrane by distinct mechanisms of action. J Clin Invest 101: 178-187

58. Tang Y-Q, Yeaman MR, Selsted ME (2002) Antimicrobial peptides from human platelets. Infect Immun 70: 6524-6533

59. Krijgsveld J, Zaat SA, Meeldijk J, van Veelen PA, Fang G, Poolman B, Brandt E, Ehlert JE, Kuijpers AJ, Engbers GH, Feijen J, Dankert J (2000) Thrombocidins, microbicidal proteins from human blood platelets, are c-terminal deletion products of $\mathrm{CXC}$ chemokines. J Biol Chem 275: 20374-20381

60. Donnelly BP, Nixon AJ, Haupt JL, Dahlgren LA (2006) Nucleotide structure of equine platelet-derived growth factor- $\mathrm{A}$ and -B and expression in horses with induced acute tendonitis. Am J Vet Res 67: 1218-1225 